\title{
icLibFuzzer: Isolated-context libFuzzer for Improving Fuzzer Comparability
}

\author{
Yu-Chuan Liang \\ National Taiwan University \\ jasonliang30115@gmail.com
}

\author{
Hsu-Chun Hsiao \\ National Taiwan University \\ hchsiao@csie.ntu.edu.tw
}

\begin{abstract}
Fuzzer is a powerful fuzzer that has helped find thousands of bugs in real-world programs. However, fuzzers that seek to compare with libFuzzer and its variants face two significant limitations. First, they are restricted to use the time-tofirst-crash metric rather than the code-coverage metric because libFuzzer will abort whenever the fuzzing target crashes. Second, even if libFuzzer in the ignore-crash mode can continue after finding a crash, it may produce wrong results for programs expecting a clean global context. Thus, fuzzers wishing to compare with libFuzzer are restricted to use carefully modified programs or programs without global-context dependency. To solve this context pollution problem and enhance comparability between libFuzzer and other fuzzers, we present a new libFuzzer mode called isolated-context mode (icLibFuzzer) that isolates the contexts of each fuzzer instance and fuzzing target, allowing to reinitialize the fuzzing target's context after each execution efficiently. To implement icLibFuzzer, we modify libFuzzer's inprocess infrastructure into a lightweight forkserver infrastructure inspired by AFL's design and propose structure packing, which speeds up the fuzzing speed by about $2 x$. We compare icLibFuzzer with four state-of-the-art fuzzers (AFL, Angora, QSYM, and Honggfuzz) using several real-world programs. The experiment result shows that icLibFuzzer outperforms these four fuzzers in most target programs after 24 hours of fuzzing and maintains the lead from 24 to 72 hours. To demonstrate that we can easily keep up with libFuzzer's updates, we upgrade icLibFuzzer to using the latest libFuzzer (from LLVM9 to LLVM11) with no change to our code base. Our preliminary evaluation hints at icLibFuzzer-LLVM11's promising improvement compared with icLibFuzzer-LLVM9 and AFL++, one of the latest fuzzers in the AFL family. We hope icLibFuzzer can serve as another baseline for fuzzing research. Our source code is available at GitHub.
\end{abstract}

\section{INTRODUCTION}

Fuzzing is a popular technique for automatically finding bugs. To fuzz a computer program, which can be a standalone executable or a library function, a fuzzing tool (called a fuzzer) feeds random inputs into the program and reports inputs that cause unexpected behaviors such as hangs or crashes. These interesting inputs can further help uncover bugs in the program.

Workshop on Binary Analysis Research (BAR) 2021

21 February 2021, Virtual

ISBN 1-891562-69-X

https://dx.doi.org/10.14722/bar.2021.23013

www.ndss-symposium.org
AFL [20] and libFuzzer [14] are two of the most successful fuzzers with different design goals and have been evolving separately for several years. AFL aims to continuously fuzz executables, while libFuzzer targets library functions and stops whenever hitting a crash. As a result, fuzzers in the AFL and libFuzzer families mainly consider the code-coverage and time-to-first-crash metrics, respectively. It is challenging to compare them directly with each other over the same metrics.

This comparability issue is solved partially by enabling the ignore-crash mode in libFuzzer. The ignore-crash mode spawns multiple fuzzer instances and fuzzes a target executable in different processes parallelly. Whenever a fuzzer instance exits or finds a crash, libFuzzer will restart the fuzzer instance. Although libFuzzer in the ignore-crash mode can fuzz continuously and restart after a crash, the fuzzing results may be incorrect when previous non-crash runs pollute the global context because of libFuzzer's in-process infrastructure. Therefore, most fuzzers were compared with libFuzzer only on few manually tuned datasets (e.g., fuzzer-test-suite [9]) that allow both fuzzer families to operate correctly.

Due to the lack of comparability, the advances in libFuzzer seem to be left unnoticed in the state-of-the-art academic papers, which predominantly consider the code-coverage metric. For example, libFuzzer has integrated advanced instrumentation features such as CMP tracing since 2017, but similar features were unavailable in AFL-based fuzzers until 2019 in RedQueen [2] (which is later integrated into AFL++ [7]). To this end, we believe a bridge between these two fuzzer families is needed to foster knowledge accumulation and sharing in the community.

In this work, we present isolated-context libFuzzer (icLibFuzzer), a new libFuzzer mode that bridges the two fuzzer families and allows them to be compared over commonly seen metrics on arbitrary programs. To solve the context pollution problem, icLibFuzzer runs in the AFL-like forkserver architecture and correctly manages the global context between executions. As speed is a crucial requirement of fuzzers, we further optimize icLibFuzzer's implementation via structure packing, achieving similar performance to other forkserverbased implementation while preserving libFuzzer's advanced instrumentation features.

To demonstrate that icLibFuzzer improves fuzzer comparability, we compare icLibFuzzer with four state-of-the-art fuzzers (AFL, Angora, Honggfuzz, and QSYM) using the 
code-coverage metric on several real-world programs. icLib-6 Fuzzer outperforms all of the tested fuzzers in most target programs after 24 hours of fuzzing. Moreover, icLibFuzzer maintains the lead and continues to increase the code coverage from 24 to 72 hours $^{1}$.

To incentivize adoption, we provide a compiler wrapper to compile standalone programs without modifying the source code. As libFuzzer is under active development and new features may appear in the future, we also provide a guideline for adding its new features into icLibFuzzer. To demonstrate that we can easily keep up with libFuzzer's updates, we upgrade icLibFuzzer to using the latest libFuzzer (i.e., upgrading from LLVM9 to LLVM11) with no change to our code base. Our preliminary evaluation hints at icLibFuzzer-LLVM11's promising improvement compared with icLibFuzzer-LLVM9 and AFL++, one of the latest fuzzers in the AFL family that integrates multiple enhanced techniques.

This paper makes the following contributions:

1) We design and implement icLibFuzzer, a new libFuzzer mode that isolates the contexts of each fuzzing target and fuzzer instance, enabling continuous fuzzing on arbitrary programs while inheriting libFuzzer's advanced instrumentation features and analysis techniques.

2) We propose the structure packing technique to improve the running speed of icLibFuzzer by roughly $2 x$.

3) We evaluate icLibFuzzer using several real-world programs and find that icLibFuzzer outperforms all of the tested fuzzers in most target programs, implying that icLibFuzzer is a competitive fuzzing tool and may serve as another evaluation baseline for fuzzing research.

4) We release our source code at https:/github.com/ csienslab/icLibFuzzer.

\section{BACKGROUND}

libFuzzer supports several advanced instrumentation features that have not been fully adopted by other fuzzers, but it is challenging to compare libFuzzer with others due to its inprocess design. We briefly introduce libFuzzer in this section and explain the comparability issues in the next section.

\section{A. libFuzzer Overview}

Similar to AFL, libFuzzer is a coverage-guided fuzzer, which collects coverage information through instrumentation, selects interesting inputs (called seeds) based on the collected information, and mutates seeds to generate new inputs.

For example, to collect program branches covered by inputs, code-coverage instrumentation inserts instructions to the target binary to record the current program counter right after each conditional branch, exemplified by the following $\mathrm{C}$ code:

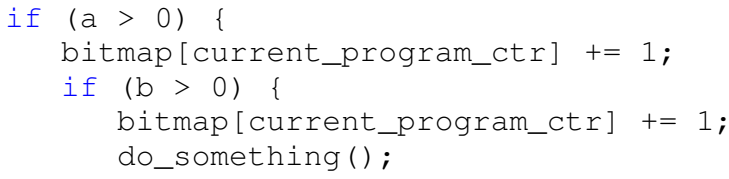

${ }^{1} 72$ hours is the longest time budget that we can afford in our experiments.
After the program terminates, the bitmap array records the branches reached by the input. Thus, given a set of inputs, we can measure a fuzzer's branch coverage (a type of codecoverage metrics) by accumulating the information recorded in the bitmap.

While AFL mainly collects code-coverage information and keeps coverage-increasing inputs as seeds, libFuzzer collects additional information such as the branch condition via instrumentation and keeps inputs that make a variety kinds of "progress" (e.g., closer to pass a conditional branch), as we will see in the next subsection.

\section{B. libFuzzer's Advanced Instrumentation Features}

libFuzzer supports several advanced instrumentation features to assist fuzzing. Some of these features are unavailable in other fuzzers or appear years later. For example, libFuzzer implemented CMP tracing in 2017, but similar ideas were unavailable in other fuzzers until 2019 in RedQueen [2].

Below we choose and describe four advanced instrumentation features in libFuzzer which may impact code coverage significantly, and have also been ported to our icLibFuzzer: CMP tracing, value profiling, compare function, and dataflow trace. We also provide a guideline in Section VII to port new libFuzzer features to icLibFuzzer.

1) CMP tracing: Due to their random nature, fuzzers often struggle to discover inputs satisfying complex branch conditions, such as $x==65536$ or $p w d=="$ secr $3 t "$. Instead of random guessing, libFuzzer supports a salient feature called CMP tracing, which hooks all of the CMP instructions (in the LLVM IR instruction set) at compile time and stores the two operands, indexed by their xored value. Take the following $\mathrm{C}$ code snippet as an example:

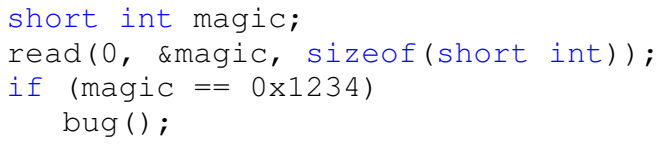

The if branch in Line 3 will be compiled into a CMP instruction, so libFuzzer inserts instrumentation code to save the compared targets:

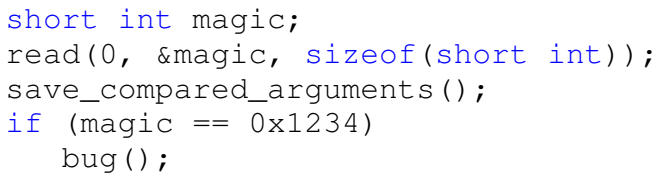

When executing Line 3, the instrumentation code will store magic and $0 \times 1234$, both indexed by magic $\oplus 0 \times 1234$. These values will then be used when mutating seeds into new inputs, such as replacing magic in input into $0 \times 1234$.

2) Value profiling: When value profiling is enabled, libFuzzer collects additional information to quantify the progress made toward passing each branch condition at runtime. Take the following code as an example: 


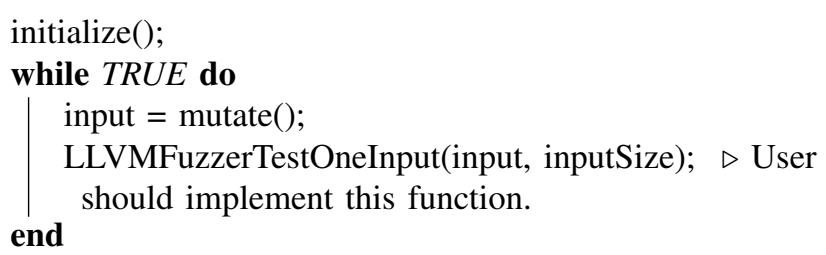

Fig. 1: libFuzzer's in-process fuzzing

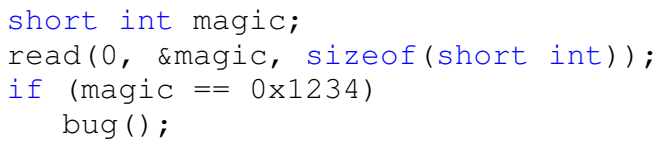

In this example, though all magic values except $0 \times 1234$ will fail the branch condition at Line 3 , some values are closer to passing the condition than others. Finding an input closer to passing the condition should be considered progress and saved as a new seed. For example, assuming that distance between two values is defined as the number of different bits, then if we mutate magic from $0 \times 1237$ to $0 \times 1235,0 \times 1235$ should be saved as a seed because $0 \times 1235$ differs from the target $(0 \times 1234)$ by one bit, while $0 \times 1237$ has a two-bit difference.

When enabling value profiling, libFuzzer uses the program counter as an index to store the distance information calculated based on the xored value of the compared operands. For example, the distance can be defined as the number of zero bits in the xored value. If the distance indexed by the same program counter reduces, which means that we are closer to solving the branch, the input will become a new seed.

3) Compare function: Besides CMP tracing for the CMP instruction, libFuzzer also hooks functions related to string comparison (e.g., strstr, memcmp, and strncasecmp), and stores the function parameters for mutation later.

4) Dataflow trace: Hooking the compare instruction and string comparison functions allows libFuzzer to retrieve the compared values, but does not reveal the relationship between the input bits and the bits of the compared value. Dataflow trace taints an input to uncover the mapping between the input and compared value by checking the taint label.

\section{C. libFuzzer's in-process infrastructure}

libFuzzer adopts an in-process infrastructure for achieving high fuzzing speed (i.e., executions per second). In an inprocess infrastructure, the fuzzer instance (libFuzzer) and the fuzzing target (LLVMFuzzerTestOneInput) work in the same context and share the same virtual memory space, as shown in Figure 1 and Figure 2. By contrast, AFL uses a forkserver infrastructure, in which each fuzzer instance and fuzzing target has an isolated context.

Despite the higher speed, the in-process infrastructure is more fragile and subject to the context pollution problem

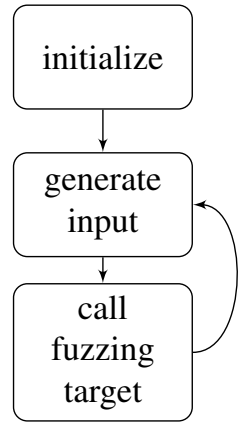

Fig. 2: libFuzzer's in-process fuzzing workflow

because the fuzzer instance may behave incorrectly due to bugs triggered in the fuzzing target, and the fuzzing target may behave incorrectly due to global context polluted in previous runs of fuzzing target.

The original libFuzzer can tolerate context pollution for two main reasons. First, it targets library functions (such as png_sig_cmp in libpng), and the fragility amplifies the impact of silent, non-crash bugs (e.g., memory leak), which are fatal in library functions. ${ }^{2}$ Second, the impact of polluted context is limited because libFuzzer stops whenever encountering a crash, and the libFuzzer user is expected to fix the bug before finding the next one.

\section{COMPARABILITy IsSUES}

This section highlights two comparability issues when prior work compares libFuzzer with other fuzzers and explains why existing solutions are insufficient. These issues motivate our design and implementation of icLibFuzzer.

\section{A. Lack support of common evaluation metrics}

Fuzzers are often evaluated by how much code they covered in a fixed time, how many bugs they found in a fixed time, and how fast they found all intended bugs. However, it is hard to measure libFuzzer's progress using these metrics on arbitrary programs because libFuzzer terminates immediately whenever the fuzzing target crashes. Moreover, the crash may be triggered by a polluted global context rather than the program's bug. Thus, previous work is either limited to using the time-to-first-crash metric [21], compared on fine-tuned datasets [1], [9], or has to enable libFuzzer's ignore-crash mode, which restarts the fuzzer right after each crash [5].

Enabling the ignore-crash mode solves this comparability issue partially but also introduces additional problems. First, restarting a fuzzer is time-consuming, thus slowing down the fuzzing process. Worst yet, restarts may frequently happen because fuzzing often triggers the same crash repeatedly. Second, the context pollution problem is less tolerable when we are interested in metrics such as code coverage and the number of bugs found because the polluted context may result

\footnotetext{
${ }^{2} \mathrm{~A}$ memory leak bug may be tolerable in a standalone program with a short lifetime because the operating system will handle memory leaks after the program terminates. However, a memory leak is fatal in library functions since the memory leak will persist and exhaust memory eventually.
} 
in an incorrect measurement of these metrics. Simply restarting the fuzzer after a crash is insufficient to avoid a broken context caused by non-crash bugs, which we will explain in the next subsection.

Some fuzzers [6], [21] avoid the context pollution problem by limiting their comparisons to a small set of manually crafted targets (e.g., Google's fuzzer-test-suite) or programs without global-context dependency, but again this limits the comparison scope.

\section{B. Incorrect results due to context pollution}

Even if libFuzzer in the ignore-crash mode can continue after finding a crash input to the fuzzing target, it may produce wrong results for targets expecting a clean global context. We identify two cases in which the ignore-crash mode may behave wrongly during fuzzing due to context pollution.

a) Programs depending on global variables: The first case is fuzzing from a main function that assumes correctlyinitialized global variables before being called.

A $\mathrm{C} / \mathrm{C}++$ program can safely assume that global variables with no initial value will be set to zero. However, when fuzzing such a program, libFuzzer treats and calls the fuzzing target like a function, so the global variables are not reinitialized when libFuzzer fuzzes the target again. Thus, if the fuzzing target modifies these global variables during its execution, the fuzzing target may act incorrectly after running a few times.

For example, getopt is a widely used function parsing the command-line arguments. It relies on a global variable optind, which indicates the next element's index in the command-line arguments:

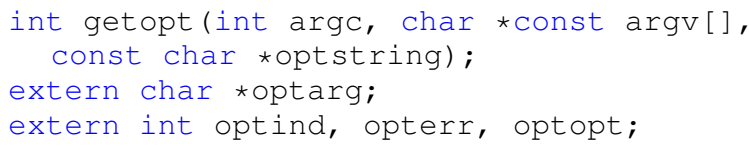

If the fuzzing target calls getopt and libFuzzer fuzzes the fuzzing target twice without reinitializing the global variable optind, getopt may behave unpredictably.

LibKluzzer [12] proposes to solve this problem by memorizing and replacing global variables every time the fuzzing target reruns. However, shared contexts other than the global variables, e.g., heap, are still polluted and will not be reset. Also, since their tool is not publicly available, it is unclear how LibKluzzer handles every possible global variable initialization, including some that can only be correctly reinitialized by calling the corresponding functions. Take the following $\mathrm{C}$ code as an example:

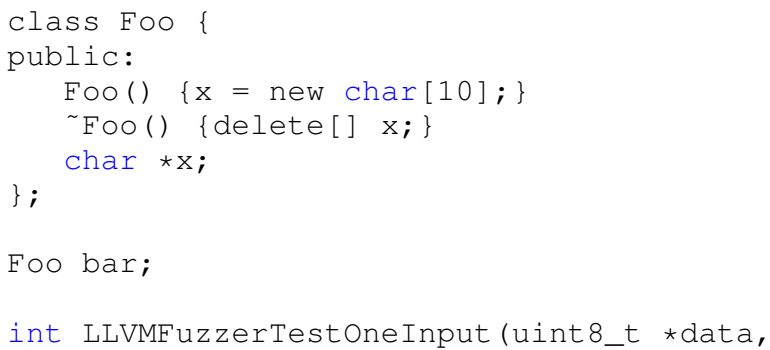

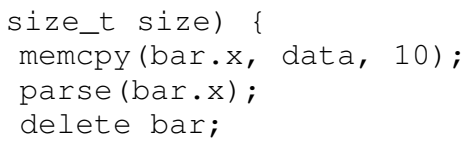

bar is a global variable. When LibKluzzer resets bar according to the previous snapshot after the function LLVMFuzzerTestone Input exits, bar. $x$ will be reinitialized to a memory address that has already been freed at line 14 . Moreover, LibKluzzer cannot handle programs with memory leak, explained below.

b) Programs with memory leak: Another form of context pollution occurs when fuzzing programs that have memory leak bugs. Since the fuzzing target runs in the same context as the fuzzer instance, if the fuzzing target contains a memory leak, the memory leak will persist and keep consuming the memory until libFuzzer crashes and restarts.

\section{ICLIBFUZZER}

This section presents icLibFuzzer's design and implementation. icLibFuzzer resolves the two comparability issues while achieving speed comparable to AFL. The key idea is to isolate each run of the fuzzing target and the fuzzer instance and implement this context isolation efficiently by changing libFuzzer's in-process infrastructure into a forkserver infrastructure. Additionally, we propose structure packing to improve fuzzing speed, set CPU affinity to ensure comparability, and provide a compiler wrapper to compile a standalone program without implementing the LLVMFuzzerTestoneInput function.

\section{A. Efficient context isolation}

There are two possible approaches to isolate context between each run of fuzzing target and fuzzing instance.

1) Naive forkserver: A straightforward yet expensive approach to ensuring a clean context would be forking from the fuzzer instance and running the fuzzing target every time. Since the fork call will spawn another process, the global context between each run of fuzzing target and fuzzing instance will be separated. However, this approach will introduce unnecessary overhead because many complicated logic and data structures in the fuzzer instance are unnecessary for the fuzzing target, and the main memory size impacts the forking speed significantly according to our experiment in Section V-D.

2) Lightweight forkserer: Inspired by AFL's infrastructure, instead of forking directly from the fuzzer instance, we can first fork and execve to create a forkserver, and fork from the forkserver. Because this approach requires less writable memory, it is faster than the native forkserver approach. The speed comparison is shown in Table V in Section V-C

Thus, we take the lightweight forkserver approach and separate the fuzzer instance into two components-main controller and forkserver. The pseudocode in Figure 3 and Figure 4 


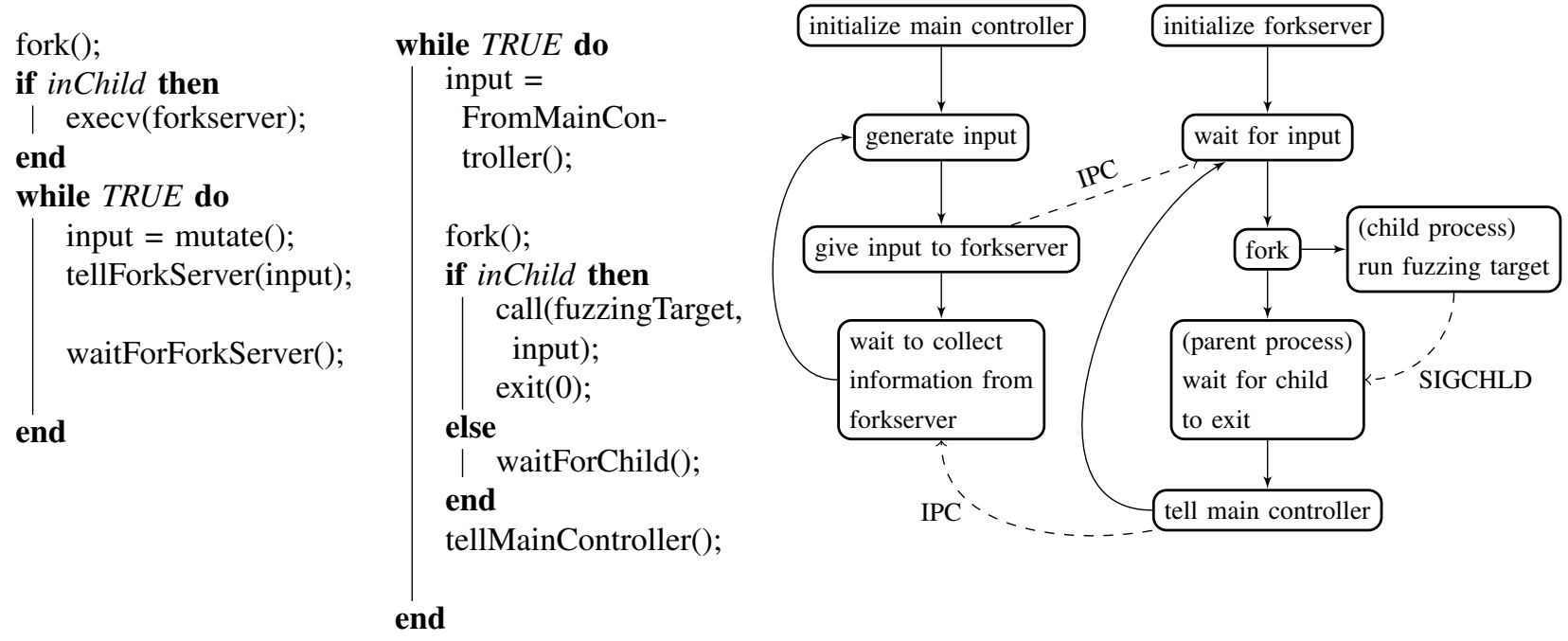

Fig. 3: icLibFuzzer: main controller
Fig. 4: icLibFuzzer: forkserver
Fig. 5: icLibFuzzer's workflow.

The dashed lines represent the communication channel. represent the main controller and forkserver, respectively, and Figure 5 illustrates how icLibFuzzer works.

The forkserver is spawned (execve after fork) from the main controller, and they communicate with each other through Inter-Process Communication (IPC). The main controller component is responsible for analyzing information collected from the instrumented program and generating inputs according to the collected information. For each input received from the main controller, the forkserver forks a child process to run the fuzzing target and collects instrumentation information. This design ensures that the fuzzing target always starts running from a clean context, free from the pollution of the previous runs, and is much faster than forking directly from fuzzing instance.

Moreover, we use shared memory as a fast IPC approach to pass instrumentation information back to the main controller from the forkserver. To achieve this, we move the data structure that stores instrumentation information into shared memory.

\section{B. Structure Packing}

Although this forkserver infrastructure is faster than forking directly from the main controller, it is still much slower than the original libFuzzer due to the resource-consuming fork procedure.

We observe that a primary factor affecting the forking speed is the writable memory size-the memory space that may be written to in the fuzzing target. Therefore, we propose structure packing to reduce the memory size and increase the forking speed.

The idea of structure packing is to pack all data collected from instrumentation into one compact structure to minimize the wasted space. For example, the following code snippet shows several data structures used in libFuzzer to store instrumentation data. The arrays MemMemWords and Table store the fuzzing target's information, collected through instrumentation at runtime.

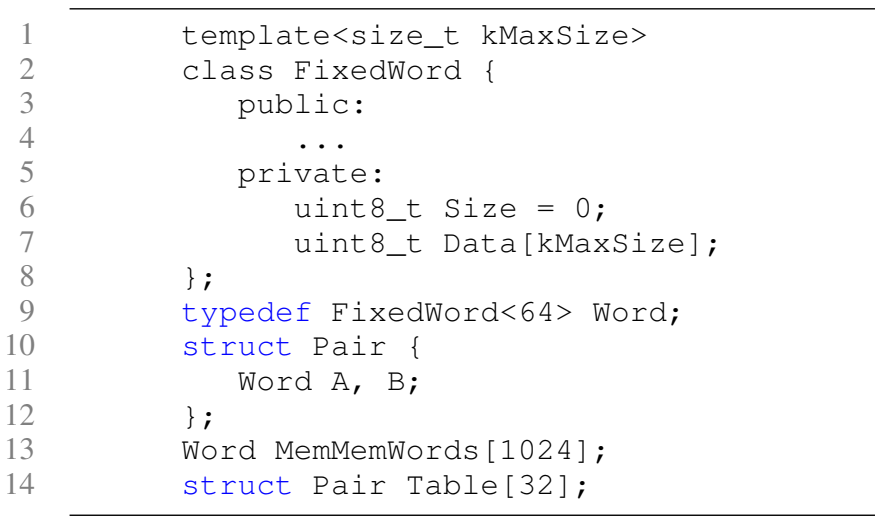

As these data structures store instrumentation information, they may be modified during the fuzzing target's execution. Instead of allocating space to them separately, icLibFuzzer packs all data into a compact structure in the shared memory to minimize the unused space:

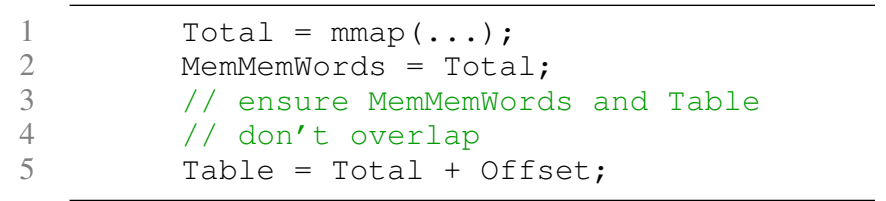

As shown in Section V-D, structure packing can double the running speed in several real-world binaries as we minimize the total memory pages that may be written to in the fuzzing target, allowing icLibFuzzer to fuzz at speed comparable to AFL.

This structure packing technique can be applied to speed up other forkserver-based fuzzers that use multiple data structures to pass information between the forkserver and main 
controller.

\section{Set CPU Affinity}

We set CPU affinity to each icLibFuzzer instance. Namely, we bind each fuzzer instance to a CPU/core. There are three reasons for doing this. First, binding a process to one CPU will improve cache locality and reduce cache miss for better performance. Second, since AFL binds its process to one CPU, most of its successors and family members do this too. Therefore, we eliminate a potential variation when comparing icLibFuzzer with fuzzers in the AFL family by following this convention. Third, binding a process to a CPU reduces interference between different fuzzing instances. Since one fuzzer instance can use at most one CPU, a fuzzer instance consisting of several processes (e.g., icLibFuzzer) will not interfere with other instances if we want to fuzz in parallel.

\section{Compiler wrapper}

icLibFuzzer comes with a compiler wrapper to automatically compile a standalone program for icLibFuzzer.

Recall that libFuzzer requires users to implement a LLVMFuzzerTestoneInput function as a callback in the fuzzer instance to identify the fuzzing target. This task can be laborious because users have to find out every input function in the fuzzing target and may accidentally introduce additional bugs. It is even more challenging when the fuzzing target reads inputs through non-standard methods or performs complex operations.

Take the following code snippet as an example:

\begin{tabular}{l}
$\operatorname{read}(0$, buf1, size1); \\
$2 \quad \operatorname{read}(0$, buf2, size $) ;$ \\
\hline
\end{tabular}

One may implement the desired LLVMFuzzerTestoneInput function as follows.

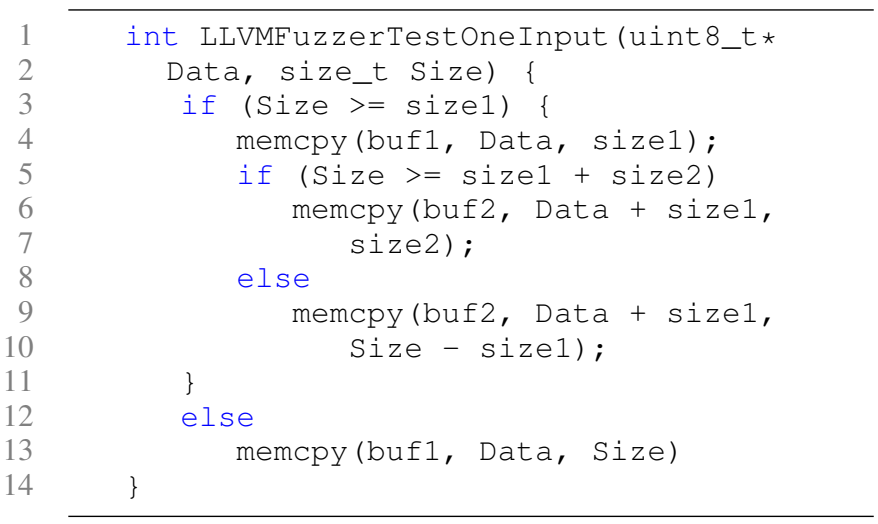

As the fuzzing target becomes more complex, the implementation of LLVMFuzzerTestoneInput may become harder and human errors may occur occasionally.

To simplify the procedure and avoid human errors, we provide a clang wrapper, clang-fast, which handles everythingjust like aft-clang-fast in AFL. All a user needs to do is compile the target project using clang-fast; no need to modify any source code manually.
TABLE I: Fuzzers used in the evaluation and their versions

\begin{tabular}{|c|c|}
\hline & version (or last-commit date) \\
\hline AFL & 2.52b (5 Nov 2017) \\
\hline Angora & 25 May 2019 \\
\hline Honggfuzz & 22 May 2019 \\
\hline libFuzzer-LLVM9 & LLVM 9 (22 April 2019) \\
\hline libFuzzer-LLVM11 & LLVM 11 (27 Sep 2020) \\
\hline QSYM & 15 October 2019 \\
\hline AFL++ & 11 Dec 2020 \\
\hline icLibFuzzer-LLVM9/11 & follow libFuzzer-LLVM9/11 \\
\hline
\end{tabular}

\section{Evaluation}

To demonstrate that icLibFuzzer improves fuzzer comparability, we compare icLibFuzzer with state-of-the-art fuzzers based on code coverage in real-world programs. The results will unveil how effective libFuzzer's advanced features are in comparison with other fuzzers. We also investigate icLibFuzzer's speed: how much is traded for comparability and how much we gain back by infrastructural and implementation optimizations.

Specifically, we ask the following three research questions:

RQ-1: Code-coverage comparison. Can icLibFuzzer achieve better code coverage compared with other state-of-theart fuzzers?

RQ-2: Execution speed difference. What is icLibFuzzer's execution speed compared with AFL, libFuzzer, and a naive forkserver implementation?

RQ-3: The effectiveness of optimization. Does structure packing improve fuzzing speed, and by how much?

\section{A. Settings}

a) Fuzzers: Table I lists the fuzzers and their versions used in our experiments. We choose AFL and Honggfuzz because they are the core fuzzers (in addition to libFuzzer) used in OSS-Fuzz [16]. Speaking more specifically, we utilize the afl-clang-fast instead of afl-gcc as the execution speed of binaries compiled by afl-clang-fast is much faster. We also choose two strong fuzzers, QSYM and Angora, released in 2019. QSYM is a hybrid fuzzer that defeats AFL and previous hybrid fuzzers. It uncovers bugs in ffmpeg which has already been fuzzed by OSS-Fuzz for years. Angora uses multiple techniques, including taint tracking, to solve branch conditions without using symbolic execution. It shows notable improvement over AFL.

Our icLibFuzzer uses the same LLVM version as libFuzzer. Throughout the evaluation, we use LLVM9 by default (i.e., icLibFuzzer-LLVM9) unless specified otherwise. We include icLibFuzzer-LLVM11 (released in late 2020) mainly to demonstrate that one can effortlessly update icLibFuzzer whenever newer versions of LLVM and libFuzzer are available. Additionally, we include AFL++, whose latest version was released in December 2020. AFL++ assembles multiple optimization techniques and integrates them into the latest AFL. It shows a significant performance boost compared with the original AFL after enabling multiple optimizations simultaneously. However, due to the limited time before the sub- 

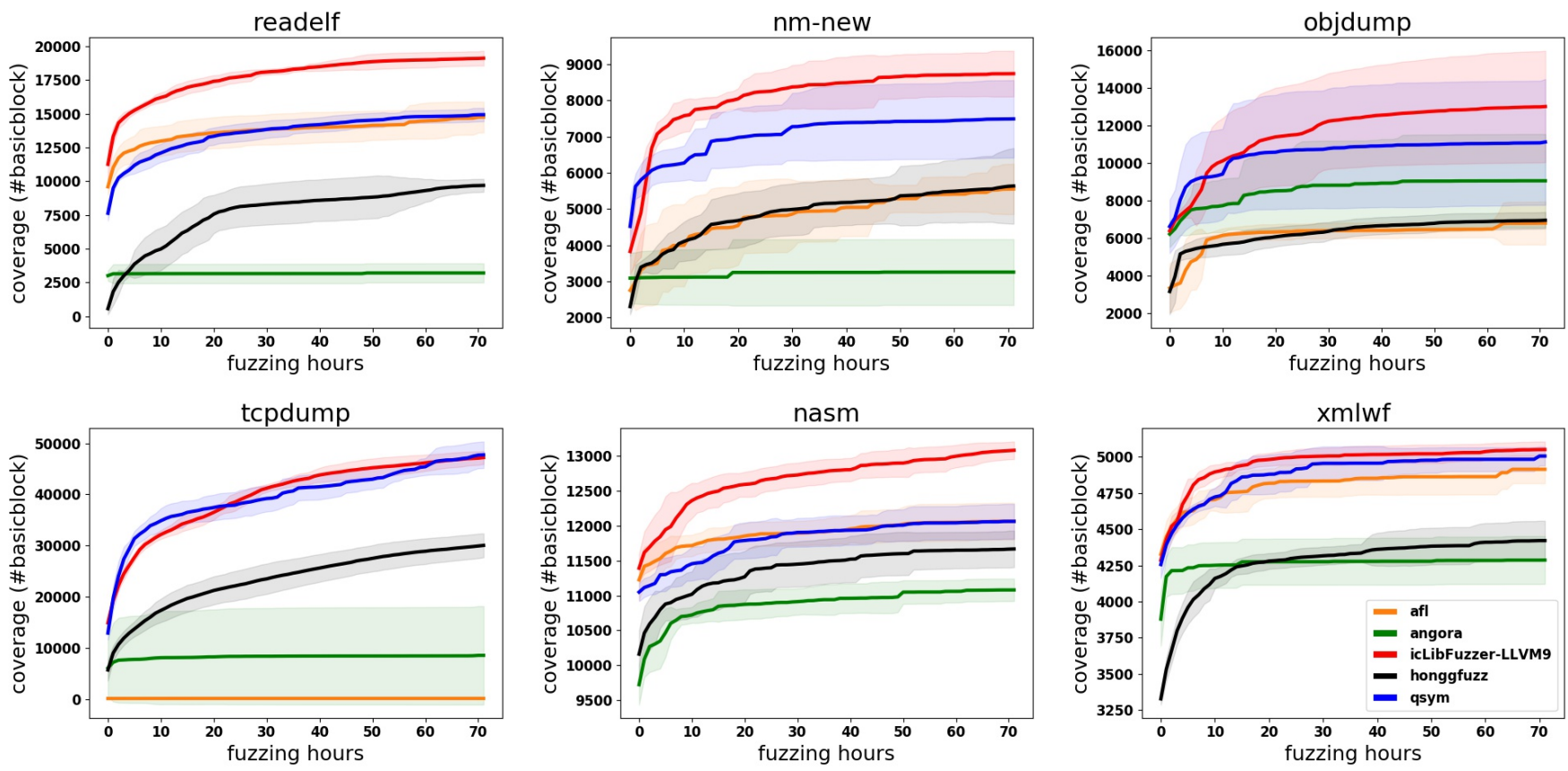

Fig. 6: Comparison between fuzzers. All the legend color mappings are the same as labeled in xmlwf.
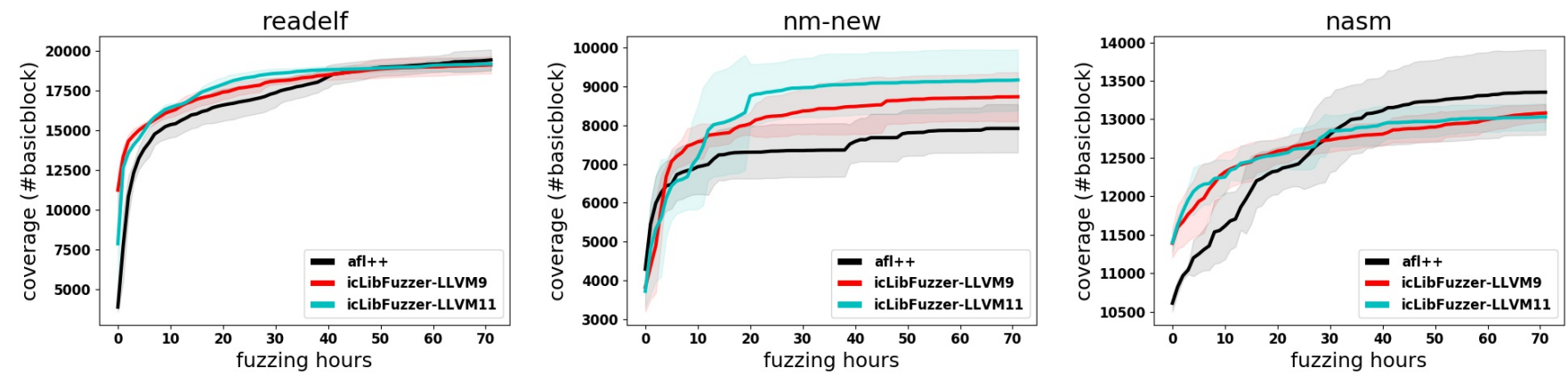

Fig. 7: Comparison among latest icLibFuzzer-LLVM11, state-of-the-art AFL++ and icLibFuzzer-LLVM9.

mission, we only compare icLibFuzzer-LLVM9, icLibFuzzerLLVM11, and AFL++ with each other, not with the other fuzzers.

b) Datasets: We use two datasets: one contains realworld programs (objdump-2.35, readelf-2.35, nm-new-2.35, tcpdump-4.9.2, xmlwf-2.2.9, nasm-2.16rc0) and the other is the fuzzer-test-suite. The real-world program dataset is for coverage and speed comparison with icLibFuzzer, while the fuzzer-test-suite is only used for speed comparison with libFuzzer.

c) Time budget: We run every binary eight times and each for 72 hours, which is the longest we can do given our resource budget. We report the average code coverage and standard deviation. Because fuzzing in the real world often lasts long (e.g., more than one month), investigating the trend after 24 hours of fuzzing can hint at the fuzzer's ability to find new code coverage continuously. d) Fuzzer configuration: To ensure fair comparisons, we assign no initial seeds to fuzzers because initial seeds may have a significant impact on fuzzing [11]. We discuss the effect of initial seeds in discussion section. We run all fuzzers with two threads to ensure a fair comparison with QSYM (which needs at least two threads). To further normalize the fuzzer's result, we calculate all the code coverage results using clang's source-based code coverage feature from interesting seeds reported by each fuzzers. We run our experiment on a machine with one AMD Ryzen Threadripper 2990WX 32Core Processor and 64GB memory, running Ubuntu 18.04 LTS.

In our evaluation, we enable the first three advanced instrumentation features introduced in Section II but not dataflow trace because dataflow trace requires manual function labeling and may provide an unfair advantage to icLibFuzzer in our comparative evaluation. 
TABLE II: Comparing each fuzzer's average speed (executions per second) on real-world programs after running 72 hours.

\begin{tabular}{|l|c|c|c|c|c|c|}
\hline & readelf & nm-new & objdump & tcpdump & nasm & xmlwf \\
\hline icLibFuzzer & 1701 & 1667 & 1030 & 1603 & 359 & 2349 \\
\hline AFL & 1708 & 1906 & 1032 & 2438 & 337 & 2983 \\
\hline qsym & 1380 & 2990 & 1174 & 3087 & 430 & 4917 \\
\hline angora & 1300 & 681 & 684 & 1068 & 419 & 1157 \\
\hline honggfuzz & 86 & 96 & 116 & 108 & 76 & 126 \\
\hline
\end{tabular}

TABLE III: Effect of structure packing. We run for 30 minutes in each cases to obtain a stable execution speed. RSS (Resident Set Size) is the RAM size consumed by the process, and exec/s stands for executions of fuzzing target per second.

\begin{tabular}{|c|c|c|c|c|}
\hline & \multicolumn{2}{|c|}{ exec/s } & \multicolumn{2}{c|}{ RSS (Bytes) } \\
& no pack & pack & no pack & pack \\
\hline objdump & 1992 & 3740 & $7260 \mathrm{~K}$ & $3636 \mathrm{~K}$ \\
\hline readelf & 2404 & 4426 & $7672 \mathrm{~K}$ & $3356 \mathrm{~K}$ \\
\hline nm-new & 2083 & 3991 & $7544 \mathrm{~K}$ & $3324 \mathrm{~K}$ \\
\hline tcpdump & 2168 & 4103 & $7504 \mathrm{~K}$ & $3492 \mathrm{~K}$ \\
\hline
\end{tabular}

TABLE IV: Comparing each fuzzer's speed (executions per second) on the fuzzer-test-suite dataset. Version of libFuzzer and icLibFuzzer here is LLVM9. We run for 30 minutes in each case to get to a relatively stable execution speed.

\begin{tabular}{|c|c|c|c|c|}
\hline & libFuzzer & icLibFuzzer & AFL & $\begin{array}{c}\text { naive } \\
\text { forkserver }\end{array}$ \\
\hline boringssl-2016-02-12 & 26707 & 1722 & 1201 & 136 \\
\hline freetype2-2017 & 9210 & 927 & 1038 & 128 \\
\hline libpng-1.2.56 & 8707 & 2556 & 3340 & 69 \\
\hline openssl-1.1.0c & 22460 & 512 & 560 & 164 \\
\hline sqlite-2016-11-14 & 20587 & 1112 & 881 & 63 \\
\hline
\end{tabular}

TABLE V: Comparing naive forkserver with lightweight forkserver. We run for 30 minutes in each case to get to a relatively stable execution speed. RSS (Resident Set Size) is the RAM size consumed by the process, and exec/s stands for executions of fuzzing target per second.

\begin{tabular}{|c|c|c|c|c|}
\hline \multirow{2}{*}{ Benchmark } & \multicolumn{2}{|c|}{ light-weight forkserver } & \multicolumn{2}{c|}{ naive forkserver } \\
& exec/s & RSS (bytes) & exec/s & RSS (bytes) \\
\hline boringssl-2016-02-12 & 1722 & $9 \mathrm{M}$ & 136 & $335 \mathrm{M}$ \\
\hline freetype2-2017 & 927 & $9 \mathrm{M}$ & 128 & $388 \mathrm{M}$ \\
\hline libpng-1.2.56 & 2556 & $6 \mathrm{M}$ & 69 & $353 \mathrm{M}$ \\
\hline openssl-1.1.0c & 512 & $11 \mathrm{M}$ & 164 & $276 \mathrm{M}$ \\
\hline sqlite-2016-11-14 & 1112 & $6 \mathrm{M}$ & 63 & $340 \mathrm{M}$ \\
\hline
\end{tabular}

\section{B. $R Q-1$}

To answer this question, we evaluate icLibFuzzer's code coverage on the real-world program dataset and compare the results with four state-of-the-art fuzzers (AFL [20], Angora [4], QSYM [19], and Honggfuzz [17]). Note that AFL, Honggfuzz, and libFuzzer have only been compared in the in-process mode (also called the persistent mode in AFL and Honggfuzz) in previous work. With icLibFuzzer, these three fuzzers can compare with each other on arbitrary programs.

Code coverage As shown in Figure 6, icLibFuzzer outperforms other fuzzers in most binaries.

We observe two interesting phenomena from the results.

1) Bad performance of Angora: Angora shows better performance than AFL in its original paper. However, we can see that Angora does not perform well in our experiment. One plausible reason may be that Angora highly relies on initial seeds, at least for these six programs we tested. Other fuzzers in our experiment seem to work reasonably despite an empty initial seed. It needs further investigation on selecting seeds wisely for Angora.
2) Code coverage after 24 hours: As suggested in [11], we should run fuzzers for 24 hours for a fair comparison. We can also see many papers [5], [7], [8] running experiments less than or equal to 24 hours. However, according to our experiment results, icLibFuzzer can still find new code coverage steadily after 24 hours, indicating that fuzzing results within 24 hours may still be insufficient to represent a fuzzer's capability to find new bugs or cover additional code.

Upgrade and compare with the latest fuzzers. To demonstrate that icLibFuzzer can easily update to incorporate newer libFuzzer versions, we update icLibFuzzer to LLVM 11 and compare it with icLibFuzzer-LLVM9 and a state-of-the-art fuzzer, AFL++ [7], released in late 2020.

As Figure 7 shows, icLibFuzzer-LLVM11 has competitive performance compared with AFL++. icLibFuzzer-LLVM11 performs better than icLibFuzzer-LLVM9 and AFL++ on $\mathrm{nm}-\mathrm{new}$. All three have similar code coverage on readelf, and AFL++ catches up after 24 hours on nasm. Due to our time budget, we can only run three binaries two times and 72 hours for each without initial seeds with these three fuzzers. We continue to run these experiments to evaluate the performance of the latest icLibFuzzer.

\section{C. $R Q-2$}

To answer this question, we run libFuzzer, icLibFuzzer, and AFL on the fuzzer-test-suite dataset and measure the number of executions per second. Using this dataset ensures libFuzzer can behave correctly.

As Table IV shows, icLibFuzzer is $4 \mathrm{x}$ to $50 \mathrm{x}$ slower than libFuzzer. However, when we compare icLibFuzzer with AFL, they run at almost the same speed even though icLibFuzzer supports more instrumentation than AFL.

We also compare the execution speed and main memory size of the naive forkserver and lightweight forkserver in Table V, as mentioned in Section IV. Since the naive forkserver approach forks directly from the fuzzer instance, it inherits the large memory space, slowing down the fork procedure and fuzzing target, thus the total execution speed of the fuzzer is $3 \mathrm{x}$ to $37 \mathrm{x}$ slower compared with icLibFuzzer.

\section{D. $R Q-3$}

As mentioned in Section IV, structure packing reduces the memory size in the forkserver and increases the speed. We 
evaluate the execution number per second with and without structure packing on four binaries, and Table III summarizes the results. On average, structure packing halves the memory usage and doubles the execution speed of icLibFuzzer.

\section{Discussion}

This section discusses two crucial factors that influence the fuzzing results: initial seeds and the number of fuzzing threads. We view them as control variables in the evaluation to ensure fair comparison among fuzzers. Further investigation is needed to understand their impact on fuzzing.

\section{A. Initial seed selection}

Prior work shows that initial seeds may affect the fuzzing result drastically [11] and recommends that it may also be a good idea to assign no initial seed (i.e., starting a fuzzer with an empty seed pool). It hypothesizes that if one fuzzer can perform well across various programs without initial seeds, it is likely that this fuzzer can also perform well on other datasets without initial seeds. Therefore, in our experiments, we fuzz these real-world programs without assigning any initial seeds. Our experiment results on real-world programs conforms with this hypothesis, but we leave choosing initial seeds wisely and fairly, and evaluating fuzzing with these initial seeds for future work.

\section{B. The number of fuzzing threads}

Several fuzzers [4], [5], [7], [13], [14] support parallelism to utilize processing power better. Additionally, when conducting fuzzing experiments, one might run multiple fuzzer instances parallelly and compute the averaged results to reduce randomness.

However, we observe from our experiment that when running multiple fuzzers in parallel, the number of concurrent fuzzing threads affects the fuzzing speed. The reason might be that due to the Linux kernel's design nature, some system calls cannot run in parallel, which limits the scalability of parallel fuzzing.

Figure 8 provides an example, showing how AFL's execution speed decrease as number of fuzzer instances increases.

This observation coincides with the results reported in prior work [18] that AFL and LibFuzzer have little performance gain when using more cores in parallel fuzzing; the total execution time per second does not increase linearly with the number of cores used.

Hence, fuzzing experiments should control the number of fuzzer instances running simultaneously. For example, if we would like to run icLibFuzzer on objdump for 30 times and compute the average code coverage, we cannot split the experiment into 10 plus 20 times; otherwise, the execution speed will differ significantly, making the average value meaningless.

\section{Case Study}

Due to our time budget, we did not fuzz many real-world programs to find bugs (e.g., enabling the address sanitizer). Among those we tested, we found one crash on nasm after fuzzing it with icLibFuzzer for 15 hours.

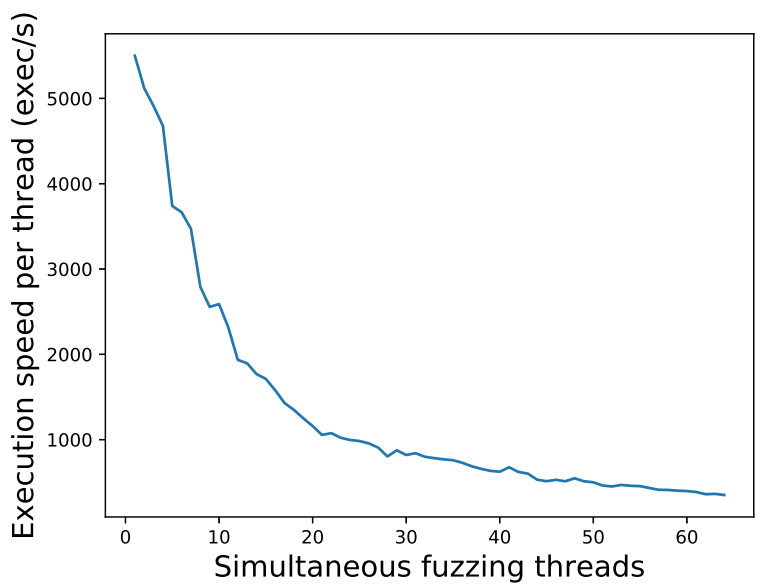

Fig. 8: Running multiple AFL fuzzer instances simultaneously. We run AFL on readelf with 1 to 64 fuzzer instances simultaneously. We spend 30 minutes in each case to wait for the execution speed to become rather stable and report the average speed.

The crash happens in the paste_tokens function:

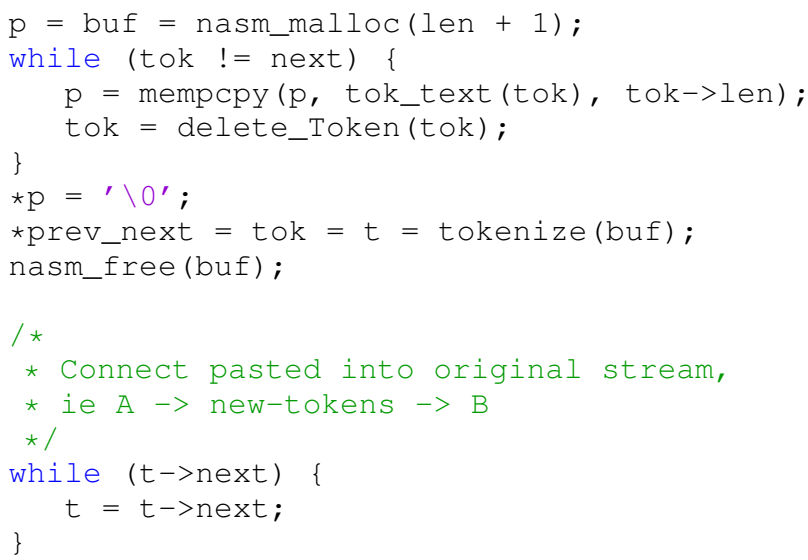

The object $t$ returned by tokenize in line 7 is a link list, and in lines 14-16 it tries to find the tail of this link list. However, it fails to check if $t$ is NULL or not. If buf in line 13 is NULL, then the returned $t$ will be NULL, causing line 14 to crash as it tries to dereference a NULL pointer.

\section{GUIDELINE FOR UPGRADING TO NEWER LIBFUZZER}

As libFuzzer evolves actively, we design icLibFuzzer to be generic enough to work with future libFuzzer updates and provide a guideline to port libFuzzer's new features to icLibFuzzer quickly.

1) Non-instrumentation features: Because the change we made in libFuzzer mainly affects how the fuzzer handles data structure that stores instrumentation information, libFuzzer updates that are irrelevant to instrumentation should work seamlessly without any modification to icLibFuzzer. 
2) Instrumentation features: icLibFuzzer separates the fuzzer instance's context and fuzzing target's context, and we use shared memory to pass the information collected from instrumentation to the main controller. To add new data structure that stores instrumentation information, simply follow the two steps below:

a) Increase the allocated memory size: The first step is increasing the allocated memory size to incorporate the new instrumentation information, but the increase should be as small as possible to minimize the impact on fuzzing speed. The member method Initialize in structure TracePC in FuzzerTracePC.h will allocate several contiguous shared memory pages storing every data structure related to instrumentation in order to share information between main controller and forkserver, One should try to minimize the required amount of increased memory because the memory size dominates icLibFuzzer's execution speed, as shown in Section V.

b) Map data structure to shared memory: After allocating additional shared memory, we point the newly allocated shared memory to the data structure storing information collected from instrumentation. When the instrumentation code in the fuzzing target gathers new information, it will be passed to the main controller by the newly added data structure on the shared memory.

\section{RELATED WORK}

Our work aims to improve comparability between libFuzzerbased fuzzers and other fuzzers in AFL family. In this section, we review related work that enhances, integrates, or compares with libFuzzer.

\section{A. Enhancing libFuzzer}

Several projects aim to improve libFuzzer [14], [15]. ParmeSan [21] implements sanitizer-oriented fuzzing on top of libFuzzer, aiming to optimize for bug coverage. FuzzerGym [6] applies reinforcement learning to improve seed mutation, implements the idea by combining OpenAI Gym with libFuzzer, and evaluates using the fuzzer-test-suite dataset.

Entropic [3] improves libFuzzer's seed scheduling algorithm. It quantifies the amount of information gained by each seed and chooses which seed to mutate first according to this value. Entropic is available in the latest libFuzzer (included in LLVM11, released in September 2020). As we show in the evaluation, icLibFuzzer can support this new libFuzzer feature easily, demonstrating the strength of our fuzzer. Moreover, icLibFuzzer-LLVM11 (using the latest libFuzzer) has competitive performance with AFL++ (v3), a powerful AFLbased fuzzer released in December 2020; this reconfirms the need to bridge the libFuzzer and AFL families, allowing new techniques to be shared transparently.

LibKluzzer [12] uses libFuzzer as a part of its hybrid fuzzer engine. To mitigate the context pollution problem, it identifies global variables, snapshots these global variables, and reinitializes these global variables in every fuzzing iteration. However, this approach may face several problems as mentioned in Section IV.

Our work differs from previous work in that we aim to improve libFuzzer's comparability with other fuzzers over a wide range of programs. Thus, we focus on solving the context pollution problem and reducing the manual efforts in modifying the target code. We also pay attention to implementation details to preserve libFuzzer's performance advantage.

\section{B. Integrating or Comparing with libFuzzer}

EnFuzz [5] combines fuzzers with diverse capabilities to increase overall code coverage, and one of the included fuzzers is libFuzzer. EnFuzz implements a monitor thread to restart libFuzzer whenever it crashes, which is similar to enabling the ignore-crash mode in libFuzzer. However, as mentioned in Section IV, EnFuzz's approach does not fully solve the comparability issue because the context may still be polluted and produce incorrect results.

OSS-Fuzz [16] is developed to find bugs in real-world programs and employs libFuzzer as one of its components. Instead of modifying libFuzzer, OSS-Fuzz modifies the fuzzing target to ensure libFuzzer works correctly.

\section{CONCLUSION}

Although libFuzzer is under active development and supports advanced techniques such as CMP tracing and value profiling, its progress and success have not been thoroughly compared against other fuzzing tools.

In this work, we propose and implement icLibFuzzer, a new libFuzzer mode that can fuzz programs not designed to run continuously, including many real-world programs, without manually modifying them. icLibFuzzer achieves higher code coverage than several state-of-the-art fuzzers on most of the real-world programs we tested. Additionally, we design icLibFuzzer to be compatible with new features added in the future. We envision icLibFuzzer can serve as another baseline in fuzzing research.

We are currently working on integrating our compiler wrapper with FuzzGen [10], an automated tool to generate LLVMFuzzerTestOneInput for a library function, so that we can run icLibFuzzer not only on standalone programs but also library functions.

We are interested in three future research directions: (1) investigating factors critical to fuzzer comparability, such as initial seed selection; (2) improving icLibFuzzer's speed via infrastructural and implementation optimizations; and (3) exploring cache-aware structure packing to eliminate misalignment, a potential side-effect of structure packing.

\section{ACKNOWLEDGMENT}

This research was supported in part by the Ministry of Science and Technology of Taiwan (MOST 110-2628-E-002002) and Delta Electronics. We gratefully thank Chun-Ying Huang, Shih-Kun Huang, Wei-Loon Mow, and Che-Yu Wu for seeding the idea and providing insightful feedback throughout the project execution. 


\section{REFERENCES}

[1] (2020) FuzzBench - Fuzzer benchmarking as a service. [Online]. Available: https://github.com/google/fuzzbench

[2] C. Aschermann, S. Schumilo, T. Blazytko, R. Gawlik, and T. Holz, "Redqueen: Fuzzing with input-to-state correspondence," in Symposium on Network and Distributed System Security (NDSS), 2019.

[3] M. Böhme, V. J. Manès, and S. K. Cha, "Boosting fuzzer efficiency: An information theoretic perspective," in Proceedings of the 28th ACM Joint Meeting on European Software Engineering Conference and Symposium on the Foundations of Software Engineering, 2020, pp. 678-689.

[4] P. Chen and H. Chen, "Angora: Efficient fuzzing by principled search," in 2018 IEEE Symposium on Security and Privacy (SP), 2018, pp. 711725.

[5] Y. Chen, Y. Jiang, F. Ma, J. Liang, M. Wang, C. Zhou, X. Jiao, and Z. Su, "Enfuzz: Ensemble fuzzing with seed synchronization among diverse fuzzers," in 28th USENIX Security Symposium (USENIX Security 19). Santa Clara, CA: USENIX Association, Aug. 2019, pp. 1967-1983. [Online]. Available: https://www.usenix.org/conference/ usenixsecurity 19/presentation/chen-yuanliang

[6] W. Drozd and M. Wagner, "Fuzzergym: A competitive framework for fuzzing and learning," 072018.

[7] A. Fioraldi, D. Maier, H. Eißfeldt, and M. Heuse, "Afl++ : Combining incremental steps of fuzzing research," in 14th USENIX Workshop on Offensive Technologies (WOOT 20). USENIX Association, Aug. 2020. [Online]. Available: https://www.usenix.org/conference/woot20/ presentation/fioraldi

[8] V. Herdt, D. Große, J. Wloka, T. Güneysu, and R. Drechsler, "Verification of embedded binaries using coverage-guided fuzzing with systemcbased virtual prototypes," in GLSVLSI, 2020.

[9] G. Inc. (2016) A set of tests (benchmarks) for fuzzing engines (fuzzers). [Online]. Available: https://github.com/google/fuzzer-test-suite/

[10] K. Ispoglou, D. Austin, V. Mohan, and M. Payer, "Fuzzgen: Automatic fuzzer generation," in 29th USENIX Security Symposium (USENIX Security 20). USENIX Association, Aug. 2020, pp. 2271-2287. [Online]. Available: https://www.usenix.org/conference/ usenixsecurity20/presentation/ispoglou

[11] G. Klees, A. Ruef, B. Cooper, S. Wei, and M. Hicks, "Evaluating fuzz testing," in Proceedings of the 2018 ACM SIGSAC Conference on Computer and Communications Security, ser. CCS '18. New York, NY, USA: Association for Computing Machinery, 2018, p. 2123-2138. [Online]. Available: https://doi.org/10.1145/3243734.3243804

[12] H. Le, LLVM-based Hybrid Fuzzing with LibKluzzer (Competition Contribution), 04 2020, pp. 535-539.

[13] J. Liang, Y. Jiang, Y. Chen, M. Wang, C. Zhou, and J. Sun, "Pafl: Extend fuzzing optimizations of single mode to industrial parallel mode," in Proceedings of the 2018 26th ACM Joint Meeting on European Software Engineering Conference and Symposium on the Foundations of Software Engineering, ser. ESEC/FSE 2018. New York, NY, USA: Association for Computing Machinery, 2018, p. 809-814. [Online]. Available: https://doi.org/10.1145/3236024.3275525

[14] libfuzzer@googlegroups.com. (2019) LibFuzzer - a library for coverage-guided fuzz testing. [Online]. Available: http://llvm.org/docs/ LibFuzzer.html

[15] K. Serebryany, "Continuous fuzzing with libfuzzer and addresssanitizer," in 2016 IEEE Cybersecurity Development (SecDev), 2016, pp. 157-157.

[16] K. Serebryany, "Oss-fuzz - google's continuous fuzzing service for open source software." Vancouver, BC: USENIX Association, Aug. 2017.

[17] R. Swiecki, "Honggfuzz: A general-purpose, easy-to-use fuzzer with interesting analysis options," 2017. [Online]. Available: https: //github.com/google/honggfuzz

[18] W. Xu, S. Kashyap, C. Min, and T. Kim, "Designing new operating primitives to improve fuzzing performance," in Proceedings of the 2017 ACM SIGSAC Conference on Computer and Communications Security, ser. CCS '17. New York, NY, USA: Association for Computing Machinery, 2017, p. 2313-2328. [Online]. Available: https://doi.org/10.1145/3133956.3134046

[19] I. Yun, S. Lee, M. Xu, Y. Jang, and T. Kim, "QSYM : A practical concolic execution engine tailored for hybrid fuzzing," in 27th USENIX Security Symposium (USENIX Security 18). Baltimore, MD: USENIX Association, Aug. 2018, pp. 745-761. [Online]. Available: https://www.usenix.org/conference/usenixsecurity18/presentation/yun

[20] M. Zalewski. American Fuzzy Lop (AFL). [Online]. Available: http://lcamtuf.coredump.cx/afl/
[21] S. Österlund, K. Razavi, H. Bos, and C. Giuffrida, "ParmeSan: Sanitizer-guided Greybox Fuzzing," in USENIX Security, Aug. 2020. [Online]. Available: Paper=https://download.vusec.net/papers/parmesan_ sec20.pdf Code=https://github.com/vusec/parmesan 